\title{
Circuit
}

Musiques contemporaines

\section{Une curieuse douzaine de Traités sur les esthétiques transtraditionnelles}

\section{Nina Polaschegg}

Volume 28, numéro 1, 2018

URI : https://id.erudit.org/iderudit/1044379ar

DOI : https://doi.org/10.7202/1044379ar

Aller au sommaire du numéro

Éditeur(s)

Circuit, musiques contemporaines

ISSN

1183-1693 (imprimé)

1488-9692 (numérique)

Découvrir la revue

Citer ce compte rendu

Polaschegg, N. (2018). Compte rendu de [Une curieuse douzaine de Traités sur les esthétiques transtraditionnelles]. Circuit, 28(1), 101-103.

https://doi.org/10.7202/1044379ar d'utilisation que vous pouvez consulter en ligne.

https://apropos.erudit.org/fr/usagers/politique-dutilisation/ 


\section{ACTUALITÉS}

\section{Une curieuse douzaine de Traités sur les esthétiques transtraditionnelles}

Compte rendu du disque Treatises on Trans-Traditional Aesthetics, Ensemble Extrakte, Sandeep Bhagwati, Dreyer Gaido 21104, 2017.

Nina Polaschegg,

traduit de l'anglais par Julie Delisle

À première vue, ce disque évoque un traité verbal et des écrits: analyses, appréciations, décisions et possiblement évaluations réciproques. Que pourrait-on attendre d'autre d'un traité musical du XXI siècle? En bref, il s'agit ici d'un aperçu du travail de recherche de l'ensemble berlinois Extrakte, dont les musiciens et musiciennes, établis depuis plusieurs années à Berlin, sont issus de plusieurs traditions musicales et socioculturelles. Sous la direction d'Elke Moltrecht et de Sandeep Bhagwati, l'ensemble a été formé en 2013 dans le but d'échanger des idées et d'arriver à une compréhension mutuelle profonde tenant compte des points de vue distincts des musiciens, mais ayant aussi pour objectif d'élaborer quelque chose à partir des composantes esthétiques et sociales de la musique. Quels sont les points communs, où se trouvent les différences les plus marquées entre les musiciens? Comment est-il possible de créer une musique dans un contexte où les clichés habituels de la world music ne permettent qu'une approche superficielle et ne donnent lieu qu'à des adaptations et à des citations au caractère presque uniquement décoratif?

Ce CD permet d'entendre les résultats de cette collaboration, qui s'est étendue sur plusieurs années et au

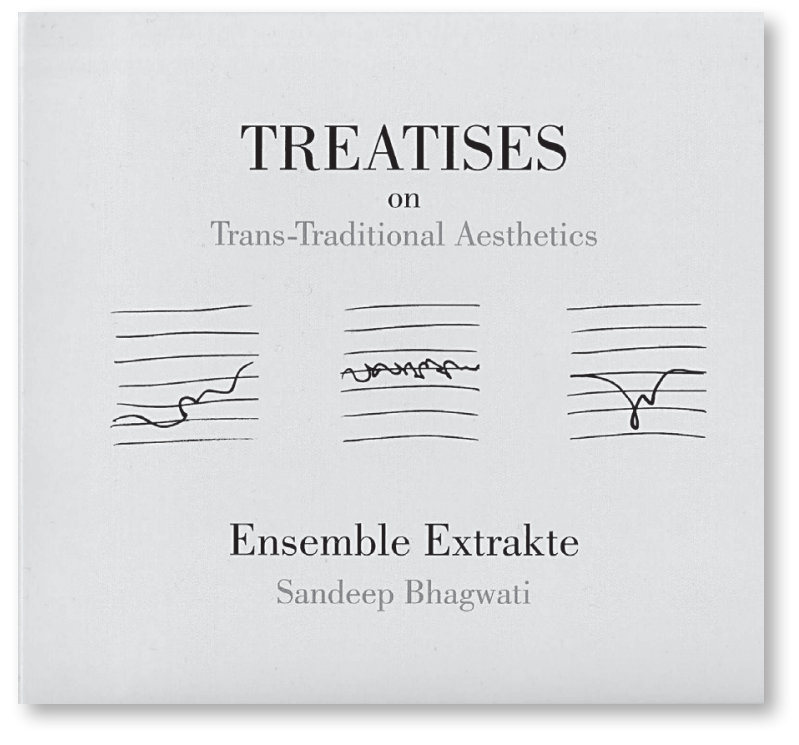

cours de laquelle les musiciens ont organisé leur travail en séances intensives de plusieurs jours. Le résultat: treize plages, treize traités constitués d'improvisations et de comprovisations réalisées à partir de concepts et d'instructions de jeu. Le tout est composé d'une alternance de miniatures en solo, de duos, de trios ainsi que de trois tutti plus longs. La plupart des concepts musicaux ont été élaborés par Sandeep Bhagwati, le directeur de l'ensemble. Celui-ci se présente comme un médiateur ou un facilitateur, dans un contexte où les décisions sont prises en groupe, afin de permettre la coexistence de différentes approches musicales. Durant les répétitions, on échange sur une variété de sujets; on joue, on se parle et on travaille à partir des 
malentendus, des points communs et des différences qui se révèlent. Résultat? Des pièces dont les titres reflètent les diverses thématiques abordées, en lien avec les interactions musicales et la communication: «De la résistance», «De la promiscuité», «De la mémoire», ou encore «De la friction». Les pièces s'appuient principalement sur des partitions graphiques élaborées en répétition, des instructions de jeu verbales et des poèmes. Dans le cas des solos, Sandeep Bhagwati a fourni des fragments de poèmes aux musiciens, qui les ont longuement travaillés pour en tirer l'essence et la traduire sous forme audible. De là, les musiciens ont développé plusieurs variations autour d'idées musicales, motiviques ou rythmiques.

Étant donné la manière dont les musiciens se sont penchés sur ces thématiques et du sérieux de leur démarche, il est difficile de donner ici un aperçu général de ces 13 traités sonores - dont les durées vont de 30 secondes à 12 minutes - d'une manière conséquente et susceptible de leur rendre justice. En fait, il faudrait au préalable donner des explications sur les dynamiques de socialisation et les expériences d'écoute, à partir desquelles des stratégies d'écoute implicites se sont développées et ont été mises en place. La perspective d'écoute de l'auteure de ce texte est celle d'une spécialiste des musiques contemporaine, jazz et ancienne, qu'elles soient improvisées ou écrites, qui privilégie une écoute inclusive et qui s'intéresse aux esthétiques transtraditionnelles. Les principes propres à la musique improvisée s'arrimant à une esthétique moderne, particulièrement dans le contexte de l'improvisation libre et conceptuelle et de la comprovisation pourraient constituer les prémisses d'une description adéquate de la musique que l'on retrouve ici.

Conséquemment, on ne s'étonnera probablement pas de l'association que j'effectue entre le Traité $n^{\circ} 1$ - intitulé «De l'insistance» et joué par la Coréenne Jin-Eun Kang au haegeum - et l'idée qui le fonde. On y reconnaît les mélismes denses et tournoyants du Vol du bourdon de Rimsky-Korsakov; une seconde lecture révèle le fragment poétique à la base de ce solo: «... des milliers d'abeilles dans ma tête...» («...tausend Bienen in meinem Kopf... »).

Le Traité $\mathrm{n}^{\circ} \mathbf{2}$ est consacré à l'idée du khali indien, le vide à la base de toute plénitude. Les musiciens se sont demandé quelle pouvait être la signification de ce concept dans chacune des cultures représentées. Dans «De la différence », on présente les idées développées à partir de ce concept, qui sont, en fin de compte, concluantes. Une trame sonore paisible, s'étendant sur toute la pièce, sert de fondement à l'ajout subséquent de gestes musicaux courts et vifs. Le tout donne lieu à une texture variée de gestes sonores évoluant sur la base d'une respiration calme.

«De la convergence» (Traité n ${ }^{0}$ 5) est un trio créé en studio à partir d'improvisations en solo. Cette pièce, comme l'explique Sandeep Bhagwati dans le livret du $\mathrm{CD}$, découle des parallèles étonnants qu'on a pu établir entre les trois miniatures.

De toute évidence, il manque ici de matériaux musicaux dans leur forme «pure», comme des structures rythmiques ou des motifs, des mélodies ou des rythmes développés sur une durée plus longue. Au lieu de concentrer l'expression sur les espaces sonores, les interactions et l'exemplification, on aurait pu intégrer différents éléments sonores, rythmiques et motiviques issus de chacune des différentes traditions dans un contexte différent.

Dans chacune des pièces, les concepts rigoureux exploités sont parfois audibles, parfois cachés. Les musiciens et musiciennes jouent de manière très organique, avec beaucoup de précision et de clarté, ainsi qu'avec intensité, respect et beaucoup de soin - parfois même très prudemment, avec un peu trop d'hésitation. De temps à autre, on a l'impression qu'ils évitent de se déranger les uns les autres ou, dit plus grossièrement, de se marcher sur les pieds. Des sonorités 
dures, des flux intenses d'énergie qui se chevauchent, de forts contrastes qui semblent s'affronter les uns les autres? De tout cela, on n'entend rien. Et lorsque dans le Traité $\mathrm{n}^{\circ}$ 4, par exemple, le contrebassiste semble s'opposer aux autres et protester avec ses notes denses et rugueuses, cela ne dure qu'un moment. Ce qui est également frappant est le fait que les techniques de jeu étendues sont à peu près absentes de l'environnement sonore, qui se limite principalement à une utilisation traditionnelle des instruments. Les timbres et les modes d'expression propres aux instruments et aux cultures musicales correspondant aux origines des musiciens ne sonnent toutefois pas comme des citations maladroites et n'apparaissent pas comme des corps étrangers.

Si on a parfois l'impression qu'une idée est déclinée de manière scolaire, c'est simplement parce que les musiciennes et musiciens ont un point de vue propre à leur démarche de recherche-création. En fait, le terme s'applique ici à un travail de recherche artistique dont le but est d'extraire les spécificités propres à chacune des cultures représentées, de travailler à partir de celles-ci et, par la suite, d'évoluer de manière plus libre et naturelle grâce aux prises de conscience effectuées et aux habiletés acquises. À ce propos, on a presque l'impression que les musiciens ont en premier lieu cherché le plus petit dénominateur commun à leurs cultures d'origine - parfois tellement différentes -, puis construit sur la base d'une certaine connaissance de l'autre avant de se diriger vers l'inconnu et la création commune de quelque chose de nouveau.

C'est dans le Traité n 13, «Du mal du pays », que l'on entend malgré tout quelques éléments « originaux ». Ils ne sont toutefois pas de nature musicale; il s'agit plutôt des voix des musiciens qui récitent, en les intégrant au jeu collectif, des fragments de poèmes dans leur langue maternelle. Une belle finale, à laquelle on peut naturellement aussi donner un sens musical. 